\title{
The mixing set with divisible capacities: a simple approach
}

\author{
Michele Conforti and Giacomo Zambelli* \\ September 2008, revised April 2009, July 2009
}

\begin{abstract}
We give a simple algorithm for linear optimization over the mixing set with divisible capacities, and derive a compact extended formulation from such an algorithm. The main idea is to apply a suitable unimodular transformation to obtain an equivalent problem that is easier to analyze.
\end{abstract}

Keywords: Mixing set, Unimodular Transformations, Dynamic Programming, Extended Formulations.

\section{Introduction}

The generalized mixing set is the set of points $(s, y) \in \mathbb{R}^{n+1}$ satisfying

$$
\begin{aligned}
s+\theta_{i} y_{i} & \geq \beta_{i} \quad i=1 \ldots, n \\
s & \geq 0 \\
y_{i} & \in \mathbb{Z} \quad i=1 \ldots, n .
\end{aligned}
$$

where $\theta_{i}, \beta_{i} \in \mathbb{Q}$ and $\theta_{i}>0, i=1 \ldots, n$. This set arises as a relaxation of lot-sizing problems (see [8] for an extensive overview). Eisenbrand and Rothvoss [3] proved that optimizing over the generalized mixing set is NPhard in general. However, cases have been studied in the literature where

*Dipartimento di Matematica Pura e Applicata, Universitá di Padova, Via Trieste 63, 35121 Padova, Italy. (conforti@math.unipd.it, giacomo@math.unipd.it) 
the optimization problem can be solved in polynomial time. Günlük and Pochet [4] gave a description of the facet-defining inequalities for the mixing set, which is the case where $\theta_{i}=1, i=1, \ldots, n$.

An extended formulation for a polyhedron $P \subset \mathbb{R}^{d}$ is a system of inequalities $F y+G z \leq h, y \in \mathbb{R}^{d}$, defining a polyhedron in the $(y, z)$ space whose projection onto the $y$-space is $P$. When $P$ is the convex hull of a mixed integer set, we say that the formulation is compact if the size of $(F, G, h)$ is bounded by some polynomial function on the size of the description of the mixed integer set (in the case of (1), a polynomial function of $n$ and of the sizes of the $\theta_{i} \mathrm{~s}$ and $\beta_{i} \mathrm{~s}$ ). Miller and Wolsey [7] gave a compact extended formulation for the mixing set.

Zhao and de Farias [11] introduced and studied the mixing set with divisible capacities, which is the case where $\theta_{i+1} / \theta_{i}$ is an integer for every $i=1, \ldots, n-1$; they characterized the vertices and rays of the mixing set with divisible capacities, and used this characterization to derive an algorithm to maximize a linear function over the set performing $O\left(n^{4}\right)$ arithmetic operations. Thus, if we denote by $C$ the largest among the encoding sizes of $\theta_{1}, \ldots, \theta_{n}, \beta_{1}, \ldots, \beta_{n}$, Zhao and De Farias' algorithm has complexity $O\left(n^{4} C\right)$. A compact extended formulation for the mixing set with divisible capacities was given by Conforti, Di Summa and Wolsey [1]; Di Summa [2] gave a new $O\left(n^{3} C\right)$ algorithm for optimizing over the mixing set with divisible capacities, while Zhao and de Farias observed, using an improvement presented in Di Summa's algorithm, that their original algorithm could be modified to run in time $O\left(n^{3} C\right)$ as well [11]. All these results on the mixing set with divisible capacities are quite involved and technical.

Here we transform the problem of optimizing over the mixing set with divisible capacities into an equivalent integer programming problem, which we refer to as the transformed problem, via a suitable unimodular transformation. The advantage is that the transformed problem appears to be simpler to study, and the results we derive are more transparent and direct. We describe an algorithm for such problem that performs $O\left(n^{2}\right)$ arithmetic operations. Since the encoding sizes of numbers in the transformed problem can be as large as $n C$, this gives an $O\left(n^{3} C\right)$ algorithm for the mixing set with divisible capacities. We then show how one can easily derive from the algorithm a compact extended formulation for the transformed problem, and consequently for the mixing set with divisible capacities. 


\section{The transformed problem}

In our treatment, the assumption that $\theta_{i}>0, i=1, \ldots, n$ can be relaxed to $\theta_{i} \geq 0$. Therefore the constraint $s \geq 0$ needs not be considered, since we may express it as $s+\theta_{n+1} y_{n+1} \geq 0$, where $\theta_{n+1}=0$ and $y_{n+1}$ is an additional integer variable that does not actually appear in the constraints.

First we observe that, in (1), we may assume that all data are integer and all variables are restricted to be integer. In fact, let $N$ be the smallest positive integer such that $N \theta_{1}, \ldots, N \theta_{n}, N \beta_{1}, \ldots, N \beta_{n}$ are integer. Let $c_{i}=N \theta_{i}$, $b_{i}=N \beta_{i}, i=1, \ldots, n$. Thus we are interested in the set $M$ of points $(s, y) \in \mathbb{R} \times \mathbb{Z}^{n}$ satisfying

$$
N s+c_{i} y_{i} \geq b_{i}, i=1 \ldots, n .
$$

Given any vertex $(\bar{s}, \bar{y})$ of $\operatorname{conv}(M)$, it is straightforward to observe that there is one of the inequalities in (1) tight at $(\bar{s}, \bar{y})$, say $\bar{s}+\theta_{j} \bar{y}_{j}=\beta_{j}$, hence $N \bar{s}=b_{j}-c_{j} \bar{y}_{j}$ is integer, since $\bar{y} \in \mathbb{Z}^{n}$. So, by the change of variable $y_{0}=N s$, (2) is equivalent to

$$
\begin{aligned}
y_{0}+c_{i} y_{i} & \geq b_{i}, \quad i=1 \ldots, n \\
y_{i} & \in \mathbb{Z} \quad i=0, \ldots, n
\end{aligned}
$$

Therefore we are interested in the following problem: given nonnegative integers $c_{1}, \ldots, c_{n}$ such that $c_{1}\left|c_{2}\right| \ldots \mid c_{n}$, integers $b_{1}, \ldots, b_{n}$, and a vector $v \in \mathbb{R}^{n+1}$, find an optimal solution to

$$
\min \left\{v^{\top} y \mid y \in \mathbb{Z}^{n+1}, y_{0}+c_{i} y_{i} \geq b_{i}, i=1 \ldots, n\right\} .
$$

We will give an algorithm to solve (4) that performs $O\left(n^{2}\right)$ arithmetic operations on numbers $c_{1}, \ldots, c_{n}, b_{1}, \ldots, b_{n}$. Thus its complexity is $O\left(n^{2}(\log N+\right.$ $C)$. Note that, if $\theta_{i}=p_{i} / q_{i}$ and $\beta_{i}=p_{n+i} / q_{n+i}, i=1, \ldots, n$, where $p_{j}$ and $q_{j}$ are coprime integers for $j=1, \ldots, 2 n$, then $N$ can be, in the worst case,

of the order of $\prod_{j=1}^{2 n} q_{j}$. Hence the size of the encoding $N p_{j} / q_{j}$ can be of the order of $n C$ in the worst case. Thus the algorithm performing $O\left(n^{2}\right)$ arithmetic operations on $c_{1}, \ldots, c_{n}, b_{1}, \ldots, b_{n}$ provides an $O\left(n^{3} C\right)$ algorithm for the mixing set with divisible capacity.

Without loss of generality, we assume that at most one among $c_{1}, \ldots, c_{n}$ is zero, hence $c_{1}, \ldots, c_{n-1}>0$ because $c_{1}\left|c_{2}\right| \ldots \mid c_{n}$. In the remainder, we denote by $C$ the $n \times(n+1)$ matrix defining the inequalities (3). 
A unimodular transformation. Consider the following $(n+1) \times(n+1)$ integral upper-triangular matrix $U=\left(u_{i j}\right)_{0 \leq i, j \leq n}$

$U=\left[\begin{array}{rrrrr}1 & c_{1} & c_{2} & \ldots & c_{n} \\ 0 & -1 & -\frac{c_{2}}{c_{1}} & \ldots & -\frac{c_{n}}{c_{1}} \\ \vdots & & -1 & & \vdots \\ & & & \ddots & -\frac{c_{n}}{c_{n-1}} \\ 0 & & \ldots & \ldots & -1\end{array}\right], \quad$ i.e. $\quad u_{i j}=\left\{\begin{aligned} 1, & i=j=0, \\ c_{j}, & i=0,1 \leq j \leq n, \\ -1, & i=j, i>0, \\ -\frac{c_{j}}{c_{i}}, & 1 \leq i<j \leq n, \\ 0, & 0 \leq j<i \leq n .\end{aligned}\right.$

Notice that $|\operatorname{det}(U)|=1$, thus the vector $x=U^{-1} y$ is integral if and only if $y$ is integral. Therefore solving (4) is equivalent to solving

$$
\min \left\{v^{\top} U x \mid x \in \mathbb{Z}^{n+1}, C U x \geq b\right\} .
$$

One can readily verify that the system $C U x \geq b$ is

$$
x_{0}+\sum_{j=1}^{i-1} c_{j} x_{j} \geq b_{i}, \quad i=1, \ldots, n .
$$

Notice that the variable $x_{n}$ does not appear in any constraint. Therefore, if we let $w^{\top}=v^{\top} U$, we may assume $w_{n}=0$, otherwise (5) is unbounded. Therefore (5) is a problem in the variables $x_{0}, \ldots, x_{n-1}$.

\section{The algorithm.}

We present an algorithm to solve problems of the form

$$
\begin{array}{ll}
\min \sum_{j=0}^{n} w_{j} x_{j} & \\
x_{0}+\sum_{j=1}^{i} c_{j} x_{j} \geq b_{i}, \quad & i=0, \ldots, n, \\
x_{j} \in \mathbb{Z} & j=0, \ldots, n,
\end{array}
$$

where $w_{0}, \ldots, w_{n} \in \mathbb{R}, c_{1}, \ldots, c_{n}$ are positive integers such that $c_{1}\left|c_{2}\right| \ldots \mid c_{n}$, and $b_{0}, \ldots, b_{n} \in \mathbb{Z}$ (note that we have changed the indices from (6) for convenience of notation). We denote by $X$ the set of points $x=\left(x_{0}, \ldots, x_{n}\right)$ in $\mathbb{Z}^{n+1}$ satisfying (7). 
We start by observing that the vectors

$$
r^{0}=\left[\begin{array}{c}
c_{1} \\
-1 \\
0 \\
\vdots \\
0 \\
0
\end{array}\right], r^{1}=\left[\begin{array}{c}
0 \\
\frac{c_{2}}{c_{1}} \\
-1 \\
\vdots \\
0 \\
0
\end{array}\right], \ldots, r^{n-1}=\left[\begin{array}{c}
0 \\
0 \\
0 \\
\vdots \\
\frac{c_{n}}{c_{n-1}} \\
-1
\end{array}\right], r^{n}=\left[\begin{array}{c}
0 \\
0 \\
0 \\
\vdots \\
0 \\
1
\end{array}\right]
$$

are all the extreme rays of $\operatorname{conv}(X)$. This is the case because the recession cone of $\operatorname{conv}(X)$ is equal to the recession cone of the feasible region of the linear relaxation of (7) (see Schrijver [9], Theorem 16.1), namely the cone $Q=\left\{r \in \mathbb{R}^{n+1} \mid r_{0}+\sum_{j=1}^{i} c_{j} r_{j} \geq 0, i=0, \ldots, n\right\}$. Any extreme ray must satisfy at equality $n$ of the $n+1$ inequalities defining $Q$. One can verify that, for $h=0, \ldots, n, r^{h} \in Q$ and it satisfies at equality all but the $h$ th inequality, hence $r^{0}, \ldots, r^{n}$ are all possible extreme rays of $\operatorname{conv}(X)$.

Thus (7) is unbounded if and only if $w^{\top} r^{j}<0$ for some $j$. Therefore throughout the rest of this section we assume $w^{\top} r^{j} \geq 0$ for $j=1, \ldots, n$. One can easily verify that such condition is equivalent to the following;

$$
w_{0} \geq \frac{w_{1}}{c_{1}} \geq \frac{w_{2}}{c_{2}} \geq \ldots \geq \frac{w_{n}}{c_{n}} \geq 0 .
$$

Lemma 1 If bounded, there exists an optimal solution $\bar{x}$ of (7) such that

$$
\left\lfloor\frac{b_{n}-b_{n-1}}{c_{n}}\right\rfloor \leq \bar{x}_{n} \leq\left\lceil\frac{b_{n}-b_{n-1}}{c_{n}}\right\rceil .
$$

Proof. First we show that, if $\bar{x}$ is an optimal solution of (7), we may assume that

$$
\bar{x}_{0}+\sum_{j=1}^{n-1} c_{j} \bar{x}_{j} \leq b_{n-1}+c_{n}-1 .
$$

If not, then the vector $\tilde{x}$ defined by $\tilde{x}_{n}=\bar{x}_{n}+1, \tilde{x}_{n-1}=\bar{x}_{n-1}-\frac{c_{n}}{c_{n-1}}, \tilde{x}_{j}=\bar{x}_{j}$ for $j=0, \ldots, n-2$, is a feasible solution of (7), and by (9) $w^{\top} \tilde{x} \leq w^{\top} \bar{x}$, hence $\tilde{x}$ is also an optimal solution.

By $(9), w_{n} \geq 0$, hence we may assume

$$
\bar{x}_{n}=\left\lceil\frac{b_{n}-\left(\bar{x}_{0}+\sum_{j=1}^{n-1} c_{j} \bar{x}_{j}\right)}{c_{n}}\right\rceil .
$$


By (10),

$$
\left\lceil\frac{b_{n}-\left(\bar{x}_{0}+\sum_{j=1}^{n-1} c_{j} \bar{x}_{j}\right)}{c_{n}}\right\rceil \geq\left\lceil\frac{b_{n}-b_{n-1}+1}{c_{n}}-1\right\rceil=\left\lfloor\frac{b_{n}-b_{n-1}}{c_{n}}\right\rfloor,
$$

while (7) implies

$$
\left\lceil\frac{b_{n}-\left(\bar{x}_{0}+\sum_{j=1}^{n-1} c_{j} \bar{x}_{j}\right)}{c_{n}}\right\rceil \leq\left\lceil\frac{b_{n}-b_{n-1}}{c_{n}}\right\rceil .
$$

Given an integer $\beta$ and an integer $k, 0 \leq k \leq n$, we denote by $P_{k}(\beta)$ the problem

$$
\begin{aligned}
& \min \sum_{j=0}^{k} w_{j} x_{j} \\
& x_{0}+\sum_{j=1}^{i} c_{j} x_{j} \geq b_{i}, \quad i=0, \ldots, k-1, \\
& x_{0}+\sum_{j=1}^{k} c_{j} x_{j} \geq \beta, \\
& x_{j} \in \mathbb{Z} \quad j=0, \ldots, k,
\end{aligned}
$$

and denote by $z_{k}(\beta)$ its optimal value. Notice that $(7)$ is the problem $P_{n}\left(b_{n}\right)$, and its optimal value is $z^{*}=z_{n}\left(b_{n}\right)$.

By Lemma 1, we may assume that, in an optimal solution to $P_{k}(\beta), x_{k}$ takes one of the following values

$$
x_{k}^{\downarrow}(\beta)=\left\lfloor\frac{\beta-b_{k-1}}{c_{k}}\right\rfloor, x_{k}^{\uparrow}(\beta)=\left\lceil\frac{\beta-b_{k-1}}{c_{k}}\right\rceil .
$$

Notice that $\beta-c_{k} x_{k}^{\downarrow}(\beta) \geq b_{k-1} \geq \beta-c_{k} x_{k}^{\uparrow}(\beta)$. Therefore:

$$
z_{k}(\beta)=\min \left\{w_{k} x_{k}^{\downarrow}(\beta)+z_{k-1}\left(\beta-c_{k} x_{k}^{\downarrow}(\beta)\right), w_{k} x_{k}^{\uparrow}(\beta)+z_{k-1}\left(b_{k-1}\right)\right\} .
$$

For $k=n, \ldots, 0$, define recursively integers $\beta_{k}^{1}, \ldots, \beta_{k}^{n-k+1}$ as follows: Let $\beta_{n}^{1}:=b_{n}$, and for $k=n-1, \ldots, 0$, let

$$
\begin{aligned}
\beta_{k}^{i} & :=\beta_{k+1}^{i}-c_{k+1} x_{k+1}^{\downarrow}\left(\beta_{k+1}^{i}\right), \quad i=1, \ldots, n-k ; \\
\beta_{k}^{n-k+1} & :=b_{k} .
\end{aligned}
$$


Given $k=n, \ldots, 0$, define recursively $\alpha_{k}^{1}, \ldots, \alpha_{k}^{n-k+1}$ as follows: Let $\alpha_{n}^{1}:=0$, and for $k=n-1, \ldots, 0$, let

$$
\begin{aligned}
\alpha_{k}^{i} & :=\alpha_{k+1}^{i}+w_{k+1} x_{k+1}^{\downarrow}\left(\beta_{k+1}^{i}\right), \quad i=1, \ldots, n-k ; \\
\alpha_{k}^{n-k+1} & :=\min _{i=1, \ldots, n-k} \alpha_{k+1}^{i}+w_{k+1} x_{k+1}^{\uparrow}\left(\beta_{k+1}^{i}\right) .
\end{aligned}
$$

Lemma 2 If bounded, the optimal value $z^{*}$ of (7) is

$$
\min _{i=1, \ldots, n+1} \alpha_{0}^{i}+w_{0} \beta_{0}^{i} \text {. }
$$

Proof. We show that, for $k=n, n-1, \ldots, 0$,

$$
z^{*}=\min _{i=1, \ldots, n-k+1} \alpha_{k}^{i}+z_{k}\left(\beta_{k}^{i}\right) .
$$

This will prove the theorem, since $z_{0}\left(\beta_{0}^{i}\right)=w_{0} \beta_{0}^{i}$ for $i=1, \ldots, n+1$.

For $k=n, \alpha_{n}^{1}=0$ and $\beta_{n}^{1}=b_{n}$. Since $z^{*}=z_{n}\left(b_{n}\right)$ by definition, (16) is trivially satisfied.

For $k=n-1, \alpha_{n-1}^{1}=w_{n} x_{n}^{\downarrow}\left(b_{n}\right), \alpha_{n-1}^{2}=w_{n} x_{n}^{\uparrow}\left(b_{n}\right), \beta_{n-1}^{1}=b_{n}-c_{n} x_{n}^{\downarrow}\left(b_{n}\right)$, and $\beta_{n-1}^{2}=b_{n-1}$, hence by $(11) z^{*}=z_{n}\left(b_{n}\right)=\min \left\{\alpha_{n-1}^{1}+z_{n-1}\left(\beta_{n-1}^{1}\right), \alpha_{n-1}^{2}+\right.$ $\left.z_{n-1}\left(\beta_{n-1}^{2}\right)\right\}$.

Let $k \in\{0, \ldots, n-1\}$. We assume that the condition (16) is satisfied for $k+1$, and we show that it holds for $k$. By (11) and by definition of $\beta_{k}^{i}$, $i=1, \ldots, n-k+1$, we have

$$
z_{k+1}\left(\beta_{k+1}^{i}\right)=\min \left\{w_{k+1} x_{k+1}^{\downarrow}\left(\beta_{k+1}^{i}\right)+z_{k}\left(\beta_{k}^{i}\right), w_{k+1} x_{k+1}^{\uparrow}\left(\beta_{k+1}^{i}\right)+z_{k}\left(\beta_{k}^{n-k+1}\right)\right\}
$$

Since we assume $z^{*}=\min _{i=1, \ldots, n-k} \alpha_{k+1}^{i}+z_{k+1}\left(\beta_{k+1}^{i}\right)$, then by $(17), z^{*}$ is the minimum among the following $2(n-k)$ numbers:

$$
\begin{array}{ll}
\alpha_{k+1}^{i}+w_{k+1} x_{k+1}^{\downarrow}\left(\beta_{k+1}^{i}\right)+z_{k}\left(\beta_{k}^{i}\right) & i=1, \ldots, n-k \\
\alpha_{k+1}^{i}+w_{k+1} x_{k+1}^{\uparrow}\left(\beta_{k+1}^{i}\right)+z_{k}\left(\beta_{k}^{n-k+1}\right) & i=1, \ldots, n-k
\end{array}
$$

By (14), the first $n-k$ numbers are $\alpha_{k}^{i}+z_{k}\left(\beta_{k}^{i}\right)$.

The minimum of the last $n-k$ numbers is

$$
z_{k}\left(\beta_{k}^{n-k+1}\right)+\min _{i=1, \ldots, n-k} \alpha_{k+1}^{i}+w_{k+1} x_{k+1}^{\uparrow}\left(\beta_{k+1}^{i}\right),
$$

which by (15) is $z_{k}\left(\beta_{k}^{n-k+1}\right)+\alpha_{k}^{n-k+1}$. Hence (16) is verified also for $k$.

Remark 3 Applying their recursive definition, the numbers $\alpha_{k}^{i}, \beta_{k}^{i}, k=$ $0, \ldots, n, i=1, \ldots, n-k+1$, can be computed in time $O\left(n^{2}\right)$. By Lemma 2, this gives an $O\left(n^{2}\right)$ an algorithm to compute the optimal value of (7). 


\section{A compact extended formulation.}

First we show that problem (7) can be formulated as a shortest path problem.

Let $D=(V, A)$ be digraph defined as follows. The nodeset is

$$
V=\left\{v_{k}^{i} \mid 0 \leq k \leq n, 1 \leq i \leq n-k+1\right\} \cup\{t\},
$$

where node $v_{k}^{i}$ represents number $\beta_{k}^{i}$ recursively defined in (12) and (13), while the node $t$ is a dummy node that represents the final state. Each arc will have a label representing a possible value of some variable.

For $k=1, \ldots, n, i=1, \ldots, n-k+1$, there are two arcs leaving node $v_{k}^{i}$, namely

- the $\operatorname{arc}\left(v_{k}^{i}, v_{k-1}^{i}\right)$ with $\rho\left(v_{k}^{i}, v_{k-1}^{i}\right)=x_{k}^{\downarrow}\left(\beta_{k}^{i}\right)$,

- the $\operatorname{arc}\left(v_{k}^{i}, v_{k-1}^{n-k+2}\right)$ with label $\rho\left(v_{k}^{i}, v_{k-1}^{n-k+2}\right)=x_{k}^{\uparrow}\left(\beta_{k}^{i}\right)$,

For $k=0$ and $i=1, \ldots, n+1$ there are $n+1 \operatorname{arcs}\left(v_{0}^{i}, t\right)$ with label $\rho\left(v_{0}^{i}, t\right)=$ $\beta_{0}^{i}$.

Let $A_{k}$ be the set of arcs in $D$ with tail in $\left\{v_{k}^{1}, \ldots, v_{k}^{n-k+1}\right\}, k=0, \ldots, n$. To each arc $a \in A_{k}$, we assign length $\ell(a)=w_{k} \rho(a)$. It follows from the construction of $D$ and the recursive definition (14),(15) that $\alpha_{k}^{i}$ is the length of a shortest path from $v_{n}^{1}$ to $v_{k}^{i}$. Therefore, by Lemma 2 , the value of the optimum solution of (7) is the length of a shortest path from $v_{n}^{1}$ to $t$, w.r.t. the length $\ell$.

The above construction immediately gives an extended formulation. Let $Z \subseteq\{0,1\}^{A}$ be the set of incidence vectors of paths from $v_{n}^{1}$ to $t$ in $D$. From the above discussion, all the vertices of $\operatorname{conv}(X)$ are of the form

$$
x_{k}=\sum_{a \in A_{k}} \rho(a) z_{a}, \quad k=0, \ldots, n
$$

where $z \in Z$. By Minkowski's theorem, any point in $\operatorname{conv}(X)$ is the sum of a convex combination of vertices of $\operatorname{conv}(X)$ and a conic combination of extreme rays of conv $(X)$. Since the extreme rays of $\operatorname{conv}(X)$ are given in (8), $\operatorname{conv}(X)$ is the set of points $x \in \mathbb{R}^{n}$ satisfying

$$
\begin{aligned}
& x_{k}=\sum_{a \in A_{k}} \rho(a) z_{a}+\sum_{j=0}^{n} \lambda_{j} r_{i}^{j}, \quad k=0, \ldots, n, \\
& z \in \operatorname{conv}(Z), \\
& \lambda \geq 0 .
\end{aligned}
$$


Since $D$ is acyclic, $\operatorname{conv}(Z)$ can be expressed by $|V|$ flow-balance inequalities plus the nonnegativity constraints, thus (18) gives an extended formulation for $\operatorname{conv}(X)$ with $O\left(n^{2}\right)$ variables, $O\left(n^{2}\right)$ constraints, and with $O\left(n^{2}\right)$ nonzero coefficients.

Clearly, this gives also an extended formulation for the set $Y=\{y \in$ $\left.\mathbb{Z}^{n+1} \mid y_{0}+c_{i} y_{i} \geq b_{i}, i=1, \ldots, n\right\}$, namely

$$
\operatorname{conv}(Y)=\left\{y \in \mathbb{R}^{n+1} \mid y=U x, x \in \mathbb{R}^{n+1},\left(x_{0}, \ldots, x_{n-1}\right) \in \operatorname{conv}(X)\right\} .
$$

The extended formulation for the mixing set with divisible capacities proposed by Conforti, Di Summa, and Wolsey [1] is based on an expansion of the continuous variable $s$ into the remainders of the divisions of $s$ by $\theta_{1}, \ldots, \theta_{n}$. The approach presented in this paper is different, since it follows from a Dynamic Programming recursion to solve the associated optimization problem. A technique to derive extended formulations from Dynamic Programming algorithm was introduced by Martin et al. [6]. Similar ideas were recently used by Faenza and Kaibel [5] to give a compact extended formulation for packing and partitioning orbitopes.

Example. To illustrate the extended formulation, let us consider the following example, where $n=3, c_{1}=3, c_{2}=6, c_{3}=12, b_{0}=-3, b_{1}=7$, $b_{2}=5, b_{3}=14$. So $X$ is the set of points $\left(x_{0}, x_{1}, x_{2}, x_{3}\right)$ satisfying

$$
\begin{array}{ll}
x_{0} & \geq-3, \\
x_{0}+3 x_{1} & \geq 7, \\
x_{0}+3 x_{1}+6 x_{2} & \geq 5, \\
x_{0}+3 x_{1}+6 x_{2}+12 x_{3} & \geq 14, \\
x_{0}, x_{1}, x_{2}, x_{3} \in \mathbb{Z} . &
\end{array}
$$

\begin{tabular}{|c|c|c|c|}
\hline$\beta_{3}^{1}=b_{3}=14$ & & & \\
\hline $\begin{array}{l}\frac{\beta_{3}^{1}-b_{2}}{c_{3}}=\frac{3}{4} \\
\beta_{2}^{1}=\beta_{3}^{1}-c_{3}\left|\frac{3}{4}\right|=14\end{array}$ & $\beta_{2}^{2}=b_{2}=5$ & & \\
\hline $\begin{array}{l}\frac{\beta_{2}^{1}-b_{1}}{c_{2}}=\frac{7}{6} \\
\beta_{1}^{1}=\beta_{2}^{1}-c_{2}\left\lfloor\frac{7}{6}\right\rfloor=8\end{array}$ & $\begin{array}{l}\frac{\beta_{2}^{2}-b_{1}}{c_{2}}=-\frac{1}{3} \\
\beta_{1}^{2}=\beta_{2}^{2}-c_{2}\left\lfloor-\frac{1}{3}\right\rfloor=11\end{array}$ & $\beta_{1}^{3}=b_{1}=7$ & \\
\hline $\begin{array}{l}\frac{\beta_{1}^{1}-b_{0}}{c_{1}}=\frac{11}{3} ; \\
\beta_{0}^{1}=\beta_{1}^{1}-c_{1}\left\lfloor\frac{11}{3}\right\rfloor=-1\end{array}$ & $\begin{array}{l}\frac{\beta_{1}^{2}-b_{0}}{c_{1}}=\frac{14}{3} \\
\beta_{0}^{2}=\beta_{1}^{2}-c_{1}\left\lfloor\frac{14}{3}\right\rfloor=-1\end{array}$ & $\begin{array}{l}\frac{\beta_{1}^{3}-b_{0}}{c_{1}}=\frac{10}{3} \\
\beta_{0}^{3}=\beta_{1}^{3}-c_{1}\left\lfloor\frac{10}{3}\right\rfloor=-2\end{array}$ & $\beta_{0}^{4}=b_{0}=-3$ \\
\hline
\end{tabular}

The following table illustrates the computation of the $\beta_{k}^{i} \mathrm{~s}$. 
The corresponding $x_{k}^{\downarrow}\left(\beta_{k}^{i}\right) \mathrm{s}$ and $x_{k}^{\uparrow}\left(\beta_{k}^{i}\right) \mathrm{s}$ are given next.

\begin{tabular}{|l|l|l|}
\hline$x_{3}^{\downarrow}\left(\beta_{3}^{1}\right)=0, x_{3}^{\uparrow}\left(\beta_{3}^{1}\right)=1$ & \multicolumn{2}{|c|}{} \\
\cline { 1 - 2 }$x_{2}^{\downarrow}\left(\beta_{2}^{1}\right)=1, x_{2}^{\uparrow}\left(\beta_{2}^{1}\right)=2$ & $x_{2}^{\downarrow}\left(\beta_{2}^{2}\right)=-1, x_{2}^{\uparrow}\left(\beta_{2}^{2}\right)=0$ & \\
\cline { 1 - 2 }$x_{1}^{\downarrow}\left(\beta_{0}^{1}\right)=3, x_{1}^{\uparrow}\left(\beta_{1}^{1}\right)=4$ & $x_{1}^{\downarrow}\left(\beta_{1}^{2}\right)=4, x_{1}^{\uparrow}\left(\beta_{1}^{2}\right)=5$ & $x_{1}^{\downarrow}\left(\beta_{1}^{3}\right)=3, x_{1}^{\uparrow}\left(\beta_{1}^{3}\right)=4$ \\
\hline
\end{tabular}

Figure 1 depicts the corresponding digraph $D$, with the labels $\rho(a)$ associated to each $\operatorname{arc} a$. The vertices of $\operatorname{conv}(X)$ correspond to the paths in $D$

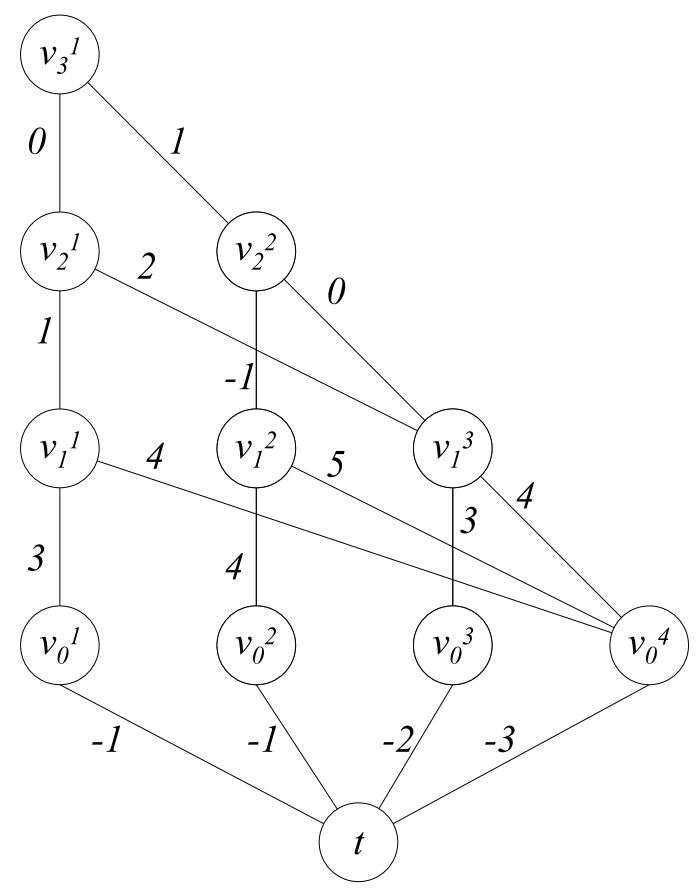

Figure 1: Auxiliary digraph with $\rho$ labels on the arcs.

from $v_{3}^{1}$ to $t$, and they are given by the labels in such paths. For example, the path $v_{3}^{1}, v_{2}^{2}, v_{1}^{2}, v_{0}^{4}, t$ corresponds to the point $(-3,5,-1,1)$, which are the labels on the edges $\left(v_{0}^{3}, t\right),\left(v_{1}^{2}, v_{0}^{3}\right),\left(v_{2}^{2}, v_{1}^{2}\right),\left(v_{3}^{1}, v_{2}^{2}\right)$, respectively. The path $\left(v_{3}^{1}, v_{2}^{1}, v_{1}^{1}, v_{0}^{1}, t\right)$ gives the point $(-1,3,1,0)$. 


\section{References}

[1] M. Conforti, M. Di Summa, L.A. Wolsey, The mixing set with divisible capacities, Proceedings of IPCO XIII, Bertinoro, Italy, (2008), 435-449.

[2] M. Di Summa, The mixing set with divisible capacities, manuscript, 2007.

[3] F. Eisenbrand, T. Rothvoss, New Hardness Results for Diophantine Approximation, manuscript, 2009.

[4] O. Günlük and Y. Pochet, Mixing mixed integer inequalities, Mathematical Programming 90 (2001), 429-457.

[5] Y. Faenza and V. Kaibel, Extended Formulations for Packing and Partitioning Orbitopes, Mathematics of Operations Research, 2008, to appear.

[6] R.K. Martin, R.L. Rardin, and B.A. Campbell, Polyhedral characterization of discrete dynamic programming, Operations Research 38 (1990), 127-138.

[7] A. Miller and L.A. Wolsey, Tight formulations for some simple MIPs and convex objective IPs, Mathematical Programming B 98 (2003), 73-88.

[8] Y. Pochet and L.A. Wolsey, Production Planning by Mixed Integer Programming, Springer Series in Operations Research and Financial Engineering, New York, 2006.

[9] A. Schrijver, Theory of Linear and Integer Programming, Wiley, New York, 1986.

[10] M. Van Vyve, A solution approach of production planning problems based on compact formulations for single-item lot-sizing models, Ph.D. thesis, Faculté des Sciences appliquées, Université catholique de Louvain, Belgium, 2003.

[11] M. Zhao and I. de Farias, The mixing-MIR set with divisible capacities, Mathematical Programming 115 (2008), 73-103. 